
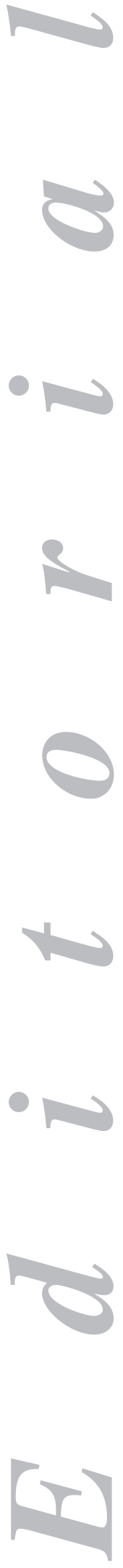

\section{Inflammatory bowel disease in Spain: problems grow}

In medical research a variety of fields diversely contribute to the understanding and eventually to the solution of problems. The relevant role played by epidemiology in this setting is clear for all of us. Specifically, its contribution in the field of inflammatory bowel disease (IBD) is of particular interest. Why is this so?

In the first place we are facing diseases whose causes have not yet been established. Albeit different authors are increasingly unveiling the pathogenesis and pathophysiology of Crohn's disease and ulcerative colitis in an elegant way, we still ignore which factor triggers the cascade of events that in both cases leads the patient to suffer from these conditions in full. A controversy on the nature of such factor divided researchers, and it still does, into geneticists and environmentalists, the latter further splitting up into those who believe that factors determining the development of the disease are purely immunologic, and those who rather claim a dietary, environmental or even microbial origin. While not detracting from the important role played by genetics, we should not lose sight that the changing, generally increasing incidence of these conditions clearly suggests that an environmental factor may collaborate with genetic predisposition in their development. In fact, this is the primary contribution of epidemiology.

Secondly, the magnitude of costs associated with inflammatory bowel disease is just beginning to be understood (1). Fortunately, drugs used in the caring of individuals diagnosed with this disease are becoming increasingly sophisticated, but their cost is also rising. From an era of unexpensive chemical therapies, their clearest representatives being glucocorticoids and azathioprine, we are entering a time of biological therapy. Its cost is presumably high, even though savings from other aspects (hospital stay, work incapacity, decreased quality of life) may likely make up for it.

Finally, let us recall that these conditions affect an increasing number of individuals who, in addition, are going through a particularly susceptible time in their lives $(1,2)$. Approximately elapsing from adolescence to first maturity, this is a time when human beings grow both personally and socially, complete their education, become integrated in groups, develop affectively, pair-bond, maybe reproduce, and end up in the complex world of labour relations; in summary, from a safe viewpoint, they are growing-up persons with an already significant capability to generate wealth. Ignorance of the number of subjects affected by these conditions will preclude our taking a first step towards quantifying the grand total of indirect expense brought forward by this ailment (1). A need for correct quantitative data should also be included in this section, which will allow us to assess the influence of inflammatory bowel disease in the quality of life of involved subjects.

In this issue of Revista Española de Enfermedades Digestivas, Rodrigo et al. (3) competently and clearly discuss the incidence of inflammatory bowel disease 
in a population area that may be deemed paradigmatic for our days: a predominantly urban, town-centered zone. Their careful study demonstrates that in that area, for two consecutive years, the adjusted incidence rate per $10^{5}$ inhabitants between 15 and 64 years of age was 9.1 for ulcerative colitis (95\% confidence interval, 5-13.1) and 7.5 for Crohn's disease (95\% confidence interval, 3.8-11.2). These rates are higher than those early reported by Cristina Saro and the coauthors of Estudio Asturiano -performed a few years before in a nearby geographic area (4)- and by many other Spanish groups, as revealed by a recent, comprehensive systematic review (5). According to the latter, in Spain, we should expect rates nearing 2 cases per year per $10^{5}$ inhabitants for Crohn's disease, and 4 cases per year per $10^{5}$ inhabitants for ulcerative colitis; even though the authors themselves emphasize that retrospective data must be cautiously interpreted in this and other contexts. In fact, Spanish estimations in the European corporate prospective study from the 1990s are somewhat higher (6). The likely present reality becomes clear as later reports by the Asturian group $(7,8)$ show higher incidences and, regarding prospective data (7), incidence rates that are virtually identical to those found by Rodrigo et al. ( 9.52 per $10^{5}$ inhabitants for ulcerative colitis and 5.95 per $10^{5}$ inhabitants for Crohn's disease).

To sum it up, the incidence of Crohn's disease and ulcerative colitis in other Asturian areas -as was already known $(7,8)$ - and in Oviedo's area -as we know it now (3)- approaches that of Northern European countries. In the face of such disquieting European convergence, the same old question of whether increased rates result from better prospection (a better access of the population to diagnostic means, greater concern for health, etc.) or from the aforementioned alleged environmental factor acting on an increasingly higher number of compatriots arises. In this respect two points should be clarified. First, let us consider that the frequency of inflammatory bowel disease, as with all diseases, increases with the thoroughness of its search. Not long ago, in an almost detective prospective study including a door-to-door survey throughout the territory of their healthcare area, some authors from India, a country with a minor incidence of inflammatory bowel disease, surprised us all with prevalence data that were much greater than those previously reported (9). Secondly, however, we should also state that Spain is no longer in the socioeconomic transition stage that characterized previous studies, and that being cared for by a physician capable of diagnosing inflammatory bowel disease has been relatively easy for a long time now (10). We are inclined to think that an actual increase in inflammatory bowel disease rates has occurred in Spain.

Other similarly interesting data were found in this study. Such is the case with the relative proportion of Crohn's disease and ulcerative colitis cases, the latter's anatomical distribution (which greatly conditions treatment), and the forms of Crohn's disease that were found according to the Vienna classification, which is becoming increasingly widespread in descriptive studies.

Finally, as the authors point out, this study is original in that it examines the incidence of inflammatory bowel disease among children. In children below 15 years of age they find an incidence of 5.7 per $10^{5}$ for Crohn's disease and of 1.4 per $10^{5}$ for ulcerative colitis, always near puberty. Unfortunately, Crohn's disease and ulcerative colitis are increasingly common in pediatric departments. No doubt, this sets a trend towards paying particular attention to this age group within epidemiologic studies on inflammatory bowel disease, which other researchers must necessarily follow into the future. 


\author{
A. López San Román and F. Bermejo ${ }^{1}$ \\ Intestinal Bowel Disease. Service or Gastroenterology. \\ Hospital Ramón y Cajal. Madrid \\ ${ }^{I}$ Service of Digestive Diseases. Hospital de Fuenlabrada. Fuenlabrada, Madrid
}

\title{
REFERENCES
}

1. Keighley MRB, Stockbrugger RW. Inflammatory bowel disease. Aliment Pharmacol Ther 2003; 18 (S3): 6670 .

2. Lashner BA, Kirsner B. The Epidemiology of inflammatory bowel disease: are we learning anything new? Gastroenterology 1992; 103: 696-8.

3. Rodrigo L, Riestra S, Niño P, Cadahía V, Tojo R, Fuentes D, et al. Incidencia de la enfermedad inflamatoria intestinal en población general en el área de Oviedo. Rev Esp Enferm Dig 2004; 96: 296-304.

4. Saro Gismerá C, Lacort Fernández M, Argüelles Fernández G, et al. Incidencia y prevalencia de la enfermedad inflamatoria intestinal en Gijón (Asturias). Gastroenterol Hepatol 2000; 23: 322-7.

5. Pajares M, Gisbert P. Epidemiología de la enfermedad inflamatoria intestinal en Espana. Una revisión sistemática. Rev Esp Enferm Dig 2001; 93: 9-21.

6. Shivananda S, Lennard-Jones J, Logan R, Fear N, Price A, Carpenter L, van Blankenstein M. Incidence of inflammatory bowel disease across Europe: is there a difference between north and south? Results of the European Collaborative Study on Inflammatory Bowel Disease (EC-IBD). Gut 1996; 39: 690-7.

7. Saro Gismera C, Riestra Menéndez S, Sánchez Fernández R, et al. Incidencia y prevalencia en enfermedad inflamatoria intestinal crónica. Estudio asturiano (EIICEA). España. An Med Interna 2003; 20 (1): 3-9.

8. Saro Gismerá C, Riestra Menéndez S, Sánchez Fernández R, et al. Epidemiología de la enfermedad inflamatoria intestinal crónica en cinco áreas de Asturias. España. An Med Interna 2003; 20 (5): 232-8.

9. Sood A, Midha V, Sood N, Bhatia AS, Avasthi G. Incidence and prevalence of ulcerative colitis in Punjab, North India. Gut 2003; 52: 1587-90.

10. González Lara V, Pérez Calle L. Epidemiología de la enfermedad inflamatoria intestinal en España. Rev Esp Enferm Dige 2001; 93: 1-8.

\section{Enfermedad inflamatoria intestinal en España: los problemas crecen}

En la investigación médica, las diferentes disciplinas contribuyen de manera diversa a la comprensión y, eventualmente, a la solución de un problema. A todos nos resulta claro el importante papel que la epidemiología juega en cualquiera de los casos. Específicamente, su aportación en el terreno de la enfermedad inflamatoria intestinal (EII) resulta de especial interés. ¿Por qué es esto así?

En primer lugar, estamos ante enfermedades de causa aún indeterminada. Si bien los diversos autores van desgranando de manera elegante la patogenia y la fisiopatología de la enfermedad de Crohn y la colitis ulcerosa, desconocemos aún el factor iniciador de la cascada de acontecimientos que, en uno y otro caso, lleva al paciente a padecer el cuadro completo de la enfermedad. La polémica acerca de la naturaleza de este factor dividió, y aún divide, a los investigadores en geneticistas y ambientalistas, y estos a su vez se dispersan entre aquellos que creen que son factores puramente inmunológicos los que determinan el desarrollo de la enfermedad, y otros que prefieren invocar un origen alimentario, ambiental o aún micro- 
biano. Sin restar importancia al papel que el terreno (la genética) juega, no podemos perder de vista que la incidencia cambiante, en general creciente, de estas enfermedades señala a las claras que un factor ambiental colabora con una predisposición genética en el desarrollo de las mismas. Y es esta la primera aportación crucial de la epidemiología.

En segundo lugar, la magnitud del coste originado por la enfermedad inflamatoria intestinal empieza ahora a ser mejor conocida (1). Los medicamentos empleados en el cuidado de las personas diagnosticadas como afectas, se van afortunadamente sofisticando, pero al tiempo su coste aumenta. De la era de los tratamientos químicos baratos, cuyos ejemplos más claros son los glucocorticoides y la azatioprina, pasamos al tiempo de la terapia biológica. El coste de esta se presume alto, aunque es probable que los ahorros en otros aspectos (tiempo de ingreso, incapacidad laboral, merma de la calidad de vida), resulten compensadores.

Finalmente, recordemos que se trata de enfermedades que afectan a un número creciente de personas, y lo hacen además en una etapa de la vida especialmente sensible $(1,2)$. Esta etapa, comprendida aproximadamente entre la adolescencia y la primera madurez, es aquella en la que el ser humano crece personal y socialmente, completa su formación, se integra en un grupo, se desarrolla afectivamente, se empareja, acaso se reproduce, y aterriza en el complejo mundo de las relaciones laborales. En suma, visto desde el lado más aséptico, una persona en formación pero ya con una capacidad importante de producir riqueza. Desconocer la cifra de personas afectadas por estos cuadros es no poder dar el primer paso para cuantificar el importe total de los gastos indirectos a los que la enfermedad va a dar lugar (1). Y debemos incluir en este apartado también la necesidad de datos cuantitativos correctos, que nos permitan evaluar la influencia de la enfermedad inflamatoria intestinal en la calidad de vida de las personas afectas.

En este número de la Revista Española de Enfermedades Digestivas, Rodrigo y cols. (3) nos exponen de una manera competente y clara el panorama de la incidencia de la enfermedad inflamatoria intestinal en un área de población, que podría considerarse paradigmática de nuestros tiempos: centrada por una ciudad media, de predominio urbano. Su cuidadosa prospección nos enseña que, en dicha zona y en 2 años consecutivos, la tasa de incidencia ajustada por $10^{5}$ habitantes entre 15 y 64 años, fue de 9,1 para la colitis ulcerosa (intervalo de confianza al 95\%, 5-13,1) y de 7,5 para la enfermedad de Crohn (intervalo de confianza al 95\%, 3,8-11,2). Estas tasas son superiores a las descritas en el trabajo inicial de Cristina Saro y los coautores del Estudio Asturiano, de cercano ámbito geográfico, unos pocos años antes (4), y de muchos otros grupos españoles, como se pone de manifiesto en una completa y reciente revisión sistemática (5). Según esta última, deberíamos esperar en España unas tasas en torno a los 2 casos por año y $10^{5}$ habitantes en la enfermedad de Crohn y de 4 casos por año y $10^{5}$ habitantes en la colitis ulcerosa, aunque los propios autores indican la precaución con la que los datos retrospectivos deben ser interpretados, en este y en otros contextos. De hecho, ya las cifras españolas del estudio prospectivo corporativo europeo de los años 90, son algo mayores (6). La probable realidad actual, queda clara cuando publicaciones posteriores del grupo asturiano $(7,8)$, muestran incidencias más altas, y en cuanto hablamos de datos prospectivos (7), tasas de incidencia prácticamente idénticas a las encontradas por Rodrigo y cols. $\left(9,52\right.$ por $10^{5}$ habitantes para la colitis ulcerosa y 5,95 por $10^{5}$ habitantes para la enfermedad de Crohn).

En suma, la incidencia de enfermedad de Crohn y de la colitis ulcerosa de otras zonas de Asturias, como ya conocíamos $(7,8)$, y del área de Oviedo, según sabemos 
ahora (3), se aproxima a la de los países del norte del continente. Cuando nos encontramos ante convergencias europeas tan intranquilizadoras como estas, la eterna pregunta es si el incremento de las tasas se debe a una mejor prospección (mejor acceso de la población a los medios diagnósticos, más preocupación por la salud, etc.), o a que el pretendido factor ambiental del que antes hablábamos actúa cada vez sobre un número mayor de compatriotas. A este respecto, cabe hacer dos matizaciones. En primer lugar, pensemos que la enfermedad inflamatoria intestinal, como todas las enfermedades, se hace más frecuente cuanto mejor se la busca. No hace mucho que unos autores de un país tan poco propenso a la enfermedad inflamatoria intestinal como es la India, nos sorprendían con datos de prevalencia mucho mayores que los previamente comunicados, en un estudio de prospección casi detectivesca, que incluyó cuestionarios pasados puerta a puerta por todo el territorio de su área sanitaria (9). Pero en segundo lugar, digamos también que España no se encuentra ya en la fase de transición socioeconómica que caracterizó a los estudios previos, y que ya hace mucho tiempo que en nuestro país es comparativamente fácil llegar a ser asistido por un médico en condiciones de hacer el diagnóstico de enfermedad inflamatoria intestinal (10). Nos inclinamos a pensar que se ha producido un aumento real de la tasa de enfermedad inflamatoria intestinal en España.

Otros datos de este estudio arrojaron igual interés. Es el caso de la proporción relativa de casos de enfermedad de Crohn y de colitis ulcerosa, las diferentes distribuciones anatómicas de esta última (que condicionan mucho el tratamiento indicado), o los tipos de enfermedad de Crohn encontrados, siguiendo la clasificación de Viena, que se va imponiendo cada vez más en estudios descriptivos.

Finalmente, y como los propios autores señalan, este estudio presenta la originalidad de investigar la incidencia de la enfermedad inflamatoria intestinal en la edad infantil. Hallan una incidencia, en menores de 15 años, de 5,7 por $10^{5}$ para la enfermedad de Crohn y de 1,4 por $10^{5}$ para la colitis ulcerosa, siempre en edades cercanas a la etapa puberal. En los Servicios de Pediatría, la enfermrdad de Crohn y la colitis ulcerosa se van haciendo, por desgracia, cada vez más habituales. Sin duda se marca así la tendencia a prestar especial atención a este sector de edad dentro de los estudios epidemiológicos sobre enfermedad inflamatoria intestinal, que otros investigadores han de seguir forzosamente en el futuro.

\title{
A. López San Román y F. Bermejo
}

Enfermedad Inflamatoria Intestinal. Servicio de Gastroenterología Hospital Ramón y Cajal. Madrid

\author{
${ }^{1}$ Servicio de Aparato Digestivo. Hospital de Fuenlabrada. Fuenlabrada, Madrid
}

